\title{
W: SPATIAL WEIGHT MATRIX DIVERSITY AND ITS IMPACT ON SPATIAL ANALYSIS RESULTS
}

\section{Simona Macková}

University of Economics, Prague, Czech Republic

Corresponding email: simona.mackova@vse.cz

\begin{abstract}
Spatial econometrics presents irreplaceable tool for regional analysis. Omitting additional information about geographical location of observed units could neglect some important influences. The spatial weight matrix $\boldsymbol{W}$ determining neighbourhood relations and degree of influence between observed units belongs to the main components of spatial analysis. Various specification approaches of this non-stochastic matrix could be applied. There is a commonly held belief that spatial regression models are sensitive to spatial weight structure. Some analytics consider it as a myth and points out incorrect interpretation of the model coefficients or misspecified models. Does it really matter what kind of specification is used? This contribution brings an empirical example of several approaches to neighbourhood specification and compares obtained results. According to findings of this analysis, especially spillover effects are incomparable. That confirms unequal performance of spatial structures. The $\boldsymbol{W}$ matrix should be built carefully at the beginning of each spatial analysis task.
\end{abstract}

Keywords: spatial analysis, spatial weight matrix, neighbourhood specification, spillover effects

\section{INTRODUCTION}

Spatial econometrics represents a very powerful approach in regional analysis of macroeconomic variables as well as in analysis on microeconomic level. Let us take a look at the Figure 1 displaying the gross domestic product per capita in EUR during the year 2014 in chosen western and central European countries. The colour range refers to the amount of GDP. According to simple observation, it is obvious that this macroeconomic variable is connected to the location and neighbouring regions can affect each other. The spatial context should not be omitted from any analysis of this kind.

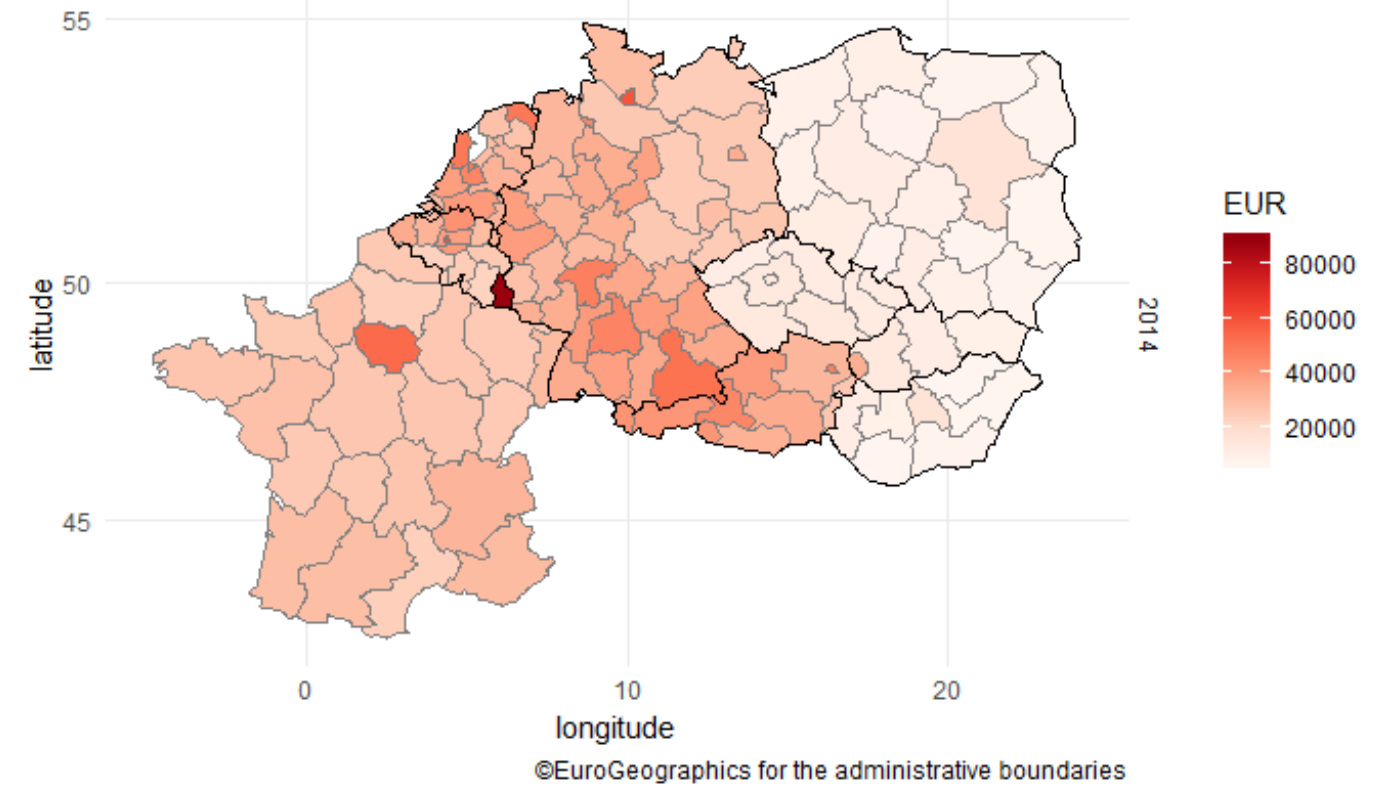

Figure 1: Distribution of GDP in western and central European countries

Reference: own elaboration

https://doi.org/10.30585/icabml-cp.v2i1.225

(C) 2018 the Authors. Production and hosting by Avicenna FZ LLC. on behalf of Dubai Business School, University of Dubai -United Arab Emirates. This is an open access article under the CC BY-NC license 
First papers considering location of observed units for economic analysis were published during the second half of the $20^{\text {th }}$ century and the interest in spatial analysis started flourishing at the turn of the millennium. (LeSage, Pace, 2009) summarizes well deeper mathematical theory of spatial econometrics. It focuses especially on methods of spatial model estimation and core theory. Basic theory for cross-section and panel data analysis as well as advanced and modern approaches could be found in often cited (Elhorst, 2014).

Contributions as (LeSage, Pace, 2014) and (Harris et al., 2011) discuss approaches of neighbourhood specification, the importance of its choice and sensitivity of models for spatial structure changes.

The spatial weight matrix $\boldsymbol{W}$ is a deterministic component of the analysis that controls behaviour of spatial dependencies. It should be defined regarding the whole environment, size and distribution of chosen units because it might influence final results and effect estimated coefficients. (LeSage, Pace, 2014) claim, that importance of neighbourhood structure is a myth in the spatial econometrics and that most of the sensitivity comes from misspecification and misinterpretation of spatial regression estimates. This paper aims to put to the test this statement.

\section{METHODOLOGY}

There is a wide choice of proposed models for spatial cross-section data analysis in (Elhorst, 2014). The full spatial model, also denoted as GNS (general nesting spatial model), considers all possible spatial interaction effects. This model is usually over-parameterized and contains too many interactions which are unnecessary. Simpler models are commonly employed.

This contribution focuses on SAR model (spatial autoregression model) where spatial interaction only with dependent variable is included. Let us denote the SAR model as follows.

$$
\boldsymbol{Y}=\delta \boldsymbol{W} \boldsymbol{Y}+\alpha \boldsymbol{\iota}_{N}+\boldsymbol{X} \boldsymbol{\beta}+\boldsymbol{\epsilon}
$$

$\boldsymbol{Y}=\left(Y_{1}, Y_{2}, \cdots Y_{N}\right)^{T}$ denotes vector of dependent variable. $\boldsymbol{X}$ stands for $N \times k$ matrix of regressors, $\boldsymbol{W}$ is $N \times N$ spatial weight matrix and $\boldsymbol{W} \boldsymbol{Y}$ an endogenous interaction. Scalar $\alpha$ is an intercept, $\boldsymbol{\beta}$ is $k \times 1$ vector of parameters and $\delta$ denotes for spatial parameter. These parameters are estimated by maximum likelihood method.

The spatial weight matrix $\boldsymbol{W}$ is a row standardized matrix. It applies $w_{i j} \in[0,1], \sum_{j=1}^{N} w_{i j}=1, i=1,2, \cdots, N$. If an element of the matrix $w_{i j}$ is equal to zero, the particular units $i$ and $j$ are not considered as neighbours. Otherwise it describes the weight of their neighbourhood relation.

One of the open questions in spatial econometric research field is how to correctly denote two units as neighbours and built a proper spatial weight matrix and whether an analyst should put effort into finding the best spatial structure.

The most intuitive approach is the contiguity method. Units with common boarders are denoted as neighbours. This method can be extended and higher order neighbours considered. The distance based method employs maximal Euclidian distance $d$ between centroids of units. A centroid can be a geographical centre, the capital city or a traffic hub of the particular unit. Other possible approach is the method of $k$-nearest neighbours that solves problem of disbalance in number of neighbours. Further inverse distance method could be considered. It is based on assumption that each unit is neighbouring all other units weighted by the distance of centroids. Quadratic or logarithmic transformations can be used to decrease or increase weights of more distant units. Let us define the off-diagonal element as $\frac{1}{d_{i j}} \operatorname{or} \frac{1}{\log \left(d_{i j}\right)}$.

All these introduced methods require so called geo-coded data - data with additional information about latitude and longitude of observed units, respectively of their centroids.

Since the spatial weight matrix $\boldsymbol{W}$ is included in regression, estimated coefficients are hardly interpretable. Direct and indirect effects are used for interpretation of results. Direct effects describe impact of change of exogenous variables to the dependent variable in the same unit. Indirect effects, also called spillovers, determine impact of change of exogenous variables in a unit to the dependent variable of its neighbouring regions. 
The effects are equal to $\left(\boldsymbol{I}_{\boldsymbol{N}}-\delta \boldsymbol{W}\right)^{-\mathbf{1}} \boldsymbol{\beta}_{\boldsymbol{k}}$, for its derivation, see (Elhorst, 2014). The diagonal elements of the matrix stand for direct effects and the off-diagonal for indirect effects. Effects are calculated for each unit separately. (LeSage, Pace, 2009) explains how to measure the overall effect.

Empirical part of this contribution applies five possible neighbourhood specification approaches on a SAR model involving macroeconomic variable: household income $(I N C)$ as the dependent variable and gross domestic product (GDP) and unemployment rate (UNE) as the explanatory variables.

$$
I N C=\alpha+\delta \boldsymbol{W I N C}+\beta_{1} \boldsymbol{G D P}+\beta_{2} \boldsymbol{U N E}+\boldsymbol{\epsilon}
$$

Observed units are regions of western and central Europe as in the Figure 1 during the year 2014. Sensitivity of model estimates, especially of estimated spillover effects is discussed. The analysis was performed in the statistical software $\mathbf{R}$ and methods of use were inspired by (Anselin, 2003) and (Bivard, 2016).

\section{FINDINGS}

Let us denote five possible spatial structures as follows:
$W_{1}$ 2-nearest neighbours
$W_{4} \frac{1}{d_{i j}}$
$W_{2}$ distance-based method with $d=180 \mathrm{~km}$
$W_{5} \frac{1}{\log \left(d_{i j}\right)}$
$W_{3}$ contiguity method

Inspired by (Formánek, Hušek, 2016), numerous models with different $k$ in $k$-nearest neighbours method were estimated and compared. The comparison is based on the Akaike informational criterion, denoted as AIC. The model with lowest AIC is chosen. In this example, $k$ equal to two performed the best. Analogical method was applied for distance based method where the most suitable maximal distance $d$ was $180 \mathrm{~km}$.

Table 1: Estimated coefficients and effects of the SAR models for various matrices $W$

\begin{tabular}{|c|c|c|c|c|c|}
\hline & $\delta$ & Direct effect & Indirect effect & AIC & \\
\hline \multirow[t]{2}{*}{$w_{1}$} & $0.623 * *$ & $0.223 * *$ & $0.261 * *$ & 2300.4 & $G D P$ \\
\hline & & $-146.438 * *$ & $-171.801 * *$ & & UNE \\
\hline \multirow[t]{2}{*}{$w_{2}$} & $0.625 * *$ & $0.220 * *$ & $0.314 * *$ & 2319.1 & $G D P$ \\
\hline & & $-159.342 * *$ & $-227.553 * *$ & & UNE \\
\hline \multirow[t]{2}{*}{$W_{3}$} & $0.459 * *$ & $0.266^{* *}$ & $0.201 * *$ & 2376.8 & $G D P$ \\
\hline & & $-206.705^{* *}$ & $-156.059 * *$ & & UNE \\
\hline \multirow[t]{2}{*}{$W_{4}$} & $-1.351 * *$ & $0.349 * *$ & $-0.202 * *$ & 2415.1 & $G D P$ \\
\hline & & $-295.115^{* *}$ & $170.425^{* *}$ & & UNE \\
\hline$W_{5}$ & $-9.448 * *$ & $0.340 * *$ & $-0.310 * *$ & 2409.8 & GDP \\
\hline
\end{tabular}




\begin{tabular}{lll}
\hline$-277.342 * *$ & $251.630 * *$ & UNE \\
\hline
\end{tabular}

** Significant at $5 \%, *$ Significant at $10 \%$

\section{Reference: own elaboration}

The first three methods brought comparable results, see Table 1 . The spatial parameter $\delta$ and the both effects are statistically significant at the confidence level of $5 \%$. With increasing GDP, one can expect increase of household income and with increase of unemployment rate, decrease of household income in the observed unit as well as in its neighbouring units. This conclusion definitely meets economical expectations.

The last two approaches performed similarly to the other approaches in sense of direct effects but completely differently regarding the indirect effects. In the fourth and fifth suggested approach, the spillovers of GDP are negative and positive for unemployment rate. This is incomparable result with the first three methods. Also all the estimates are statistically significant.

According to these results, first method of two nearest neighbours would be chosen as the most suitable. The obtained estimates meet economical expectation and the Akaike informational criterion is the lowest which points out the appropriateness of the model.

\section{CONCLUSIONS}

This contribution briefly summarized the basic theory of neighbourhood specification and determination of the $\boldsymbol{W}$ matrix. Suggested approaches were described and applied on macroeconomic variables for western and central European countries.

Based on the empirical part, statements from the article (LeSage, Pace, 2014) could be confirmed: While looking at one particular approach, stable estimates are found, even if the parameters change, e.g. number of nearest neighbours. On the other hand, the spatial structure has a strong impact on final results and its variants do not deliver equal results. The main benefits of spatial analysis are the spillovers, impacts of changes on neighbouring units. Particularly the indirect effects are very sensitive to the neighbourhood specification.

Therefore, the spatial weight matrix $\boldsymbol{W}$ was proved to be an extremely important feature of the spatial analysis and has to be defined carefully. Strong distortion of results might appear when the neighbourhood relations are specified incorrectly.

\section{REFERENCES}

Anselin L. (2003). An Introduction to Spatial Regression Analysis in R. Illinois: Urbana-Champaign.

Retrieved from http://labs.bio.unc.edu/buckley/documents/anselinintrospatregres.pdf

Bivard R. (2016). Creating Neighbours. CRAN - R Project,

Retrieved from http://cran.r-project.org/web/packages/spdep/vignettes/nb.pdf.

Elhorst J.P. (2014). Spatial econometrics: from cross-section data to spatial panel. Heidelberg: Springer.

Formánek T. \& Hušek R. (2015). The Czech Republic and its neighbors: Analysis of spatial macroeconomic dynamics. In 33th International Conference Mathematical Methods in Economics 2015, 190-195.

Retrieved from https://mme2015.zcu.cz/downloads/MME 2015 proceedings.pdf

Formánek T. \& Hušek, R. (2016). On the stability of spatial econometric models: Application to the Czech Republic and its neighbors. In 34th International Conference Mathematical Methods in Economics 2016, 213-218.

Retrieved from

http://mme2016.tul.cz/conferenceproceedings/mme2016 conference_proceedings.pdf 
Harris R., Moffat, J. \& Kravtsova V. (2011). In Search of W. Spatial Econometric Analysis, 6(3), 249-270. https://doi.org/10.1080/17421772.2011.586721

LeSage J. \& Pace R. K. (2009). Introduction to Spatial Econometrics. U.S.A.: CRC Press.

LeSage J. \& Pace R. K. (2014). The biggest myth in spatial econometrics. Econometrics, 2(4), 217-249. http://dx.doi.org/10.2139/ssrn.1725503 